\title{
PREDICTION OF PARTICULATE CONTAMINATION \\ ON APERTURE WINDOW
}

\author{
Aleck L. Lee ${ }^{*}$ and Michael C. Fong ${ }^{+}$ \\ Space Systems Division \\ Lockheed Missiles \& Space Company \\ Sunnyvale, CA 94086
}

\begin{abstract}
This paper presents an analysis to predict the effects of light scattering by surface particles on the sensor window of a missile during ascent flight. The particulate contaminant distribution on the window is calculated by tallying the number of particles in a set of size ranges. The particulate contamination at the end of the mission is predicted by adding the contributions from the events of ground and flight operations. The surface particle redistribution caused by vibroacoustically induced surface acceleration was found to contribute the most to the particulate surface contamination. The analytical surface obscuration calculation with a set of particle counts was compared to the results of image analyzer measurement. The analytical results, which were calculated with a given function of particle shape depending on the size, were more conservative than the measurement. A scattering calculation using a verified BSDF model showed that the scattering was less than 0.001 at $20^{\circ}$ off the direction of the incident light in the mid IR wavelength when the surfaces were at Level 300 initially.
\end{abstract}

\section{INTRODUCTION}

The aperture window of a tactical missile is highly contamination sensitive. The light scattering from the contaminant on the window surface contributes to the background stray light level that can be detrimental to the seeker operations. As the main effect of the contamination is light scattering when the window is exposed to the sunlight, the requirement can be also posed by the bidirectional scattering distribution function (BSDF). In this study the BSDF of the aperture window is calculated from the predicted surface particulate contamination distribution.

The service life of an aperture window starts from the delivery of the seeker hardware and ends with the impact of the target. The life span includes various phases of manufacturing and assembling process, storage, transportation, and ascent flight. This study presents a systematic approach to predict the level of particulate contamination of the aperture window in terms of obscuration increases on the aperture window during the manufacturing process and the ascent flight till the end of life.

The aperture window is located on the forecone of a tactical missile. The window has a field of view spanning from the forward direction to the side of the vehicle. A shroud, which has a smaller half-cone angle, is attached to the vehicle near the base of the forecone. The shroud protects the forecone from aeroheating during ascent, but proves to be a major contributor to the increase of particulate contamination on the aperture window.

The shroud consists of two halves with seam lines extending from the apex of the shroud to the base of the forecone assembly. One of the seam lines lies directly over the aperture window. There is a gap at the joining section of the body. The gap is sealed with an O-ring. The O-ring is sized for a $0.5 \mathrm{~mm}$ compression. Under load during ascent flight, the seam may open and ingest particles in the atmosphere to the interior of the shroud.

\footnotetext{
${ }^{*}$ Staff Engineer, IES Senior Member $\quad+$ Technical Consultant 
At deployment, the shroud opens at the seam lines. The two halves of the shroud are pushed apart by inflating the gas bags above the forecone. As the shrouds are further apart, the hinge at the base will release the shroud from the launch vehicle. The particles generated from the surfaces of the gas bags and the seam lines will fall onto the forecone and the aperture window.

The sources and particulate contamination transport mechanisms are: particle redistribution caused by surface acceleration excited by the vibroacoustic field during ascent from the shroud, ingestion of exterior particulate materials through the seam line during ascent, particles generated from activation of separation air bags, and particles generated from shroud seam line during shroud separation. Each issue is discussed in this study, and the increase of surface obscuration due to each source is predicted. The effects of the final contamination level on the window are calculated with a BSDF model.

\section{SURFACE PARTICLES AND CLEANLINESS LEVEL}

The correlation between surface particle obscuration and BRDF prediction has been investigated before (Refs. 1 and 2). For this analysis, the particle distribution data were obtained from four measurements for a sample with an image analyzer (IA). The samples were exposed to the environment of a typical optics laboratory for some time. The IA results showed the particle counts for given particle size ranges, i.e., the size bins, and the obscuration ratios under visible light and specific instrument setting conditions. The obscuration is expressed as percent area coverage (PAC). Table 1 summarizes the measured data.

Table 1 Measured data from an image analyzer.

\begin{tabular}{lrrrr}
\hline \hline $\begin{array}{l}\text { Case Number } \\
\text { Size bin, } \mu \mathrm{m}\end{array}$ & 1 & 2 & 3 & 4 \\
\hline & & & & \\
$10-25$ & 492 & 477 & 367 & 350 \\
$25-50$ & 861 & 852 & 501 & 518 \\
$50-100$ & 305 & 311 & 138 & 140 \\
$100-150$ & 43 & 38 & 20 & 21 \\
$150-250$ & 4 & 4 & 2 & 2 \\
$250-500$ & 1 & 0 & 0 & 0 \\
$500-750$ & 1 & 1 & 0 & 0 \\
$>750$ & 0 & 0 & 0 & 0 \\
\hline Measured PAC, $\%$ & 0 & 0 & 0 & 0 \\
\hline \hline
\end{tabular}

The particle counts and the percent obscuration ratios (\%OR) calculated with the PAC model are summarized in Table 2 . Table 3 shows a comparison of the measured obscuration and the computed obscuration. The obscuration measured directly with the IA is approximately $63 \%$ of the computed obscuration made with the PAC model. The discrepancies in \%OR results are attributed to the choice of particle geometry in the model and the settings of the IA scanning, such as the contrast threshold, pixel size, and so on. The last two columns of the table are the equivalent cleanliness levels calculated from the calculated \%OR value and the IA measured value. The differences in the analytical results and the IA measurements show that the analytical results tend to be more conservative. 
Table 2 Summary of measured data in numbers per sq $\mathrm{ft}$.

\begin{tabular}{crrrr}
\hline \hline $\begin{array}{c}\text { Case Number } \\
\text { Size bin, } \mu \mathrm{m}\end{array}$ & $\begin{array}{r}1 \\
\left(\# / \mathrm{ft}^{2}\right)\end{array}$ & $\begin{array}{c}2 \\
\left(\# / \mathrm{ft}^{2}\right)\end{array}$ & $\begin{array}{r}3 \\
\left(\# / \mathrm{ft}^{2}\right)\end{array}$ & $\begin{array}{r}4 \\
\left(\# / \mathrm{ft}^{2}\right)\end{array}$ \\
\hline$<10$ & 126981 & 123109 & 376493 & 359053 \\
$10-25$ & 222216 & 219894 & 513959 & 531398 \\
$25-50$ & 78718 & 80266 & 141569 & 143621 \\
$50-100$ & 11098 & 9807 & 20517 & 21543 \\
$100-150$ & 1032 & 1032 & 2052 & 2052 \\
$150-250$ & 258 & 0 & 0 & 0 \\
$250-500$ & 258 & 258 & 0 & 0 \\
$500-750$ & 0 & 0 & 0 & 0 \\
$>750$ & 0 & 0 & 0 & 0 \\
\hline Calculated \%OR & 0.1243 & 0.1182 & 0.2257 & 0.2314 \\
\hline \hline
\end{tabular}

Table 3 Comparison of obscuration by IA and PAC.

\begin{tabular}{lccccc}
\hline \hline Case & \%OR, PAC & \%OR, IA & IA/PAC & \multicolumn{2}{c}{ Equiv. Level based on } \\
$\#$ & Analytical & Measured & $\%$ & Computed & IA \\
\hline 1 & 0.1243 & 0.0792 & 63.72 & 448 & 408 \\
2 & 0.1182 & 0.0781 & 66.07 & 444 & 407 \\
3 & 0.2257 & 0.1423 & 63.05 & 505 & 461 \\
4 & 0.2314 & 0.1426 & 61.62 & 508 & 461 \\
\hline \hline
\end{tabular}

\section{SURFACE PARTICULATE CONTAMINATION INCREASE}

Particulate contamination on the aperture window increases during its service life. The sources of contamination are analyzed quantitatively in this section.

\section{Redistribution Caused by Vibroacoustic Environment}

Particles on a surface can be resuspended if the surface acceleration overcomes the adhesion force between the particle and the surface. As a launch vehicle is ascending, the vibroacoustic field generated by the booster rockets and the boundary shearing noise excite the surfaces of the launch vehicle. The surface acceleration due to vibroacoustic excitation can be calculated with analytical models, such as VAPEPS (Ref. 3). When the surface acceleration is given in power spectral density (PSD), the root mean square surface acceleration can be calculated with an integration over the spectrum to obtain the results in g-rms. The fraction of resuspended particles can be correlated to g-rms with respect to particle sizes (Ref. 4).

Since the surface acceleration is not determined at the time of the analysis, a conservative value of $12 \mathrm{~g}$-rms has been assumed. The particles resuspended from the inner side of the shroud are assumed to be uniformly distributed on the forecone. The effects of venting is not considered in this preliminary analysis. The particles on the aperture window are assumed originally to stay 
on the outside surface. This assumption may be justified by the fact that the aperture window is facing the acceleration vector. Any particle resuspended by surface acceleration would settle back to the window surface. The initial particle distribution for both the shroud and the forecone surfaces is assumed to follow that described in MIL-STD $1246 \mathrm{~B}$ with a slope of -0.926 . This distribution represents a surface which has just been cleaned, in contrast to a surface collecting particles from fallout, where the slope would be smaller, $\sim-0.3$. The effects of particle redistribution on the aperture window have been predicted and summarized in the table below.

Table $4 \quad$ Particle redistribution from shroud to aperture window due to vibroacoustic excitation.

\begin{tabular}{cccc}
\hline \hline $\begin{array}{c}\text { Shroud } \\
\text { Level }\end{array}$ & $\begin{array}{c}\text { Original } \\
\text { Level }\end{array}$ & $\begin{array}{c}\text { Final Window } \\
\text { Level }\end{array}$ & $\begin{array}{c}\text { Final } \\
\text { Obscuration (\%) }\end{array}$ \\
\hline 500 & 400 & 474 & 0.1852 \\
400 & 400 & 431 & 0.1159 \\
350 & 400 & 418 & 0.0993 \\
300 & 400 & 409 & 0.0897 \\
\hline 500 & 300 & 437 & 0.1243 \\
400 & 300 & 370 & 0.0557 \\
350 & 300 & 344 & 0.0394 \\
300 & 300 & 325 & 0.0299 \\
\hline \hline
\end{tabular}

Table 4 shows that, if the aperture window was at Level 400 per Mil-Std 1246B initially, the surface obscuration will become $0.1852 \%$ if the shroud is at Level $500,0.1159 \%$ if the shroud is at Level 400 , and so on. The obscuration of a Level 400 surface is $0.0805 \%$ and a Level 300 surface is $0.0208 \%$. If the budget of $0.25 \%$ obscuration is divided equally between the inside and outside surfaces, the obscuration allowance for each side is $0.125 \%$. The particle redistribution from a Level 500 shroud would use up all of the budget. If the exterior surface of the aperture window is at Level 400 , the cleanliness level of the shroud should be at Level 400 or better.

\section{Particulate Ingestion through Shroud Gap}

As the vehicle ascends through the atmosphere, the aerodynamic loading and the stress may cause the shroud to deform and result in a gap in the seam lines. Assuming a maximum gap size of 10 -mil, or $0.254 \mathrm{~mm}$, the total area of the seam gap is calculated to be $123 \mathrm{~mm}^{2}$. The projected area perpendicular to the flight direction is $21.4 \mathrm{~mm}^{2}$ or $0.033 \mathrm{in}^{2}$.

The amount of particulate mass, $M$, flowing through the shroud gap area in each altitude range can be calculated by

$$
\mathrm{M} \cong(\mathrm{A})(\mathrm{DL})(\mathrm{LWC} \text { or } \rho)
$$

where $A$ is the projected area of the gap perpendicular to the flight direction and DL is the traveled distance in each altitude range. LWC is the liquid water content, and $\rho$ is the density of particles in the atmosphere. The number of particles contained in a given mass $\mathbf{M}$ can be calculated with the average mass of the particles. 
The calculated results of mass and number of particles for each particle type ingested into the shroud in each altitude range are tabulated in Table 5. It is seen that relatively few rain, snow, or ice crystal particles are ingested into the shroud; but the total number of volcano dust particles can be as high as $2.9 \times 10^{7}$, based on a 10-mil gap size assumption.

Table 5 Mass and number of particles ingested into shroud through a 10- $\mu \mathrm{m}$ gap.

\begin{tabular}{cccccccc}
\hline \hline $\begin{array}{c}\text { Range } \\
\text { No. }\end{array}$ & $\begin{array}{c}\text { Altitude } \\
\text { Range } \\
(\mathrm{km})\end{array}$ & $\begin{array}{c}\text { Particle } \\
\text { Type }\end{array}$ & $\begin{array}{c}\text { Particle } \\
\text { Size } \\
(\mathrm{mm})\end{array}$ & $\begin{array}{c}\text { Density } \\
\left(\mathrm{gm} / \mathrm{cm}^{3}\right)\end{array}$ & $\begin{array}{c}\text { Distance } \\
\text { Traveled } \\
(\mathrm{m})\end{array}$ & $\begin{array}{c}\text { Mass per } \\
\text { Particle } \\
(\mathrm{gm})\end{array}$ & $\begin{array}{c}\text { No. of } \\
\text { Particles } \\
\text { Ingested }\end{array}$ \\
\hline I & $0-2$ & Rain & 1.5 & 0.03 & 4900 & $1.77 \times 10^{-3}$ & 2 \\
II & $0-2.44$ & Dust & 0.0075 & $1 \times 10^{-5}$ & 6000 & $1.21 \times 10^{-10}$ & 5792 \\
III & $2-4$ & Snow & 1.5 & 0.05 & 4800 & $1.77 \times 10^{-3}$ & 3 \\
IV & $4-7$ & Snow & 0.5 & 0.03 & 7200 & $6.54 \times 10^{-5}$ & 71 \\
V & $7-10$ & Ice Crystal & 0.3 & 0.02 & 7200 & $1.41 \times 10^{-5}$ & 218 \\
VI & $12.2-15.2$ & Volc Dust & 0.001 & $1 \times 10^{-4}$ & 7200 & $5.24 \times 10^{-13}$ & $2.9 \times 10^{7}$ \\
\hline \hline
\end{tabular}

The ingested particles are assumed to deposit on the surface of forecone uniformly. Based on this assumption, the contribution to obscuration increase from each altitude range are computed. Table 6 summarizes the results. The range numbers in the table correspond to the particle types given in Table 5. The most significant contribution to surface PAC is that from volcano dusts (Range Number VI), which amounts to $0.0094 \%$. The results show that the increases of surface contamination due to ingested particles from exterior sources may be negligible.

Table 6 Contribution to obscuration from exterior sources.

\begin{tabular}{ccccc}
\hline \hline $\begin{array}{c}\text { Range } \\
\text { Number }\end{array}$ & $\begin{array}{c}\text { No of } \\
\text { Particles }\end{array}$ & $\begin{array}{c}\text { No. Particles } \\
\text { per } \mathrm{ft}^{2}\end{array}$ & $\begin{array}{c}\text { Average } \\
\text { Size }\end{array}$ & $\begin{array}{c}\text { Contribution to } \\
\text { Obscuration (\%) }\end{array}$ \\
\hline I & 2 & 0.767 & $1.5 \mathrm{~mm}$ & 0.0014 \\
II & 5792 & 2222 & $7.5 \mu \mathrm{m}$ & 0.0001 \\
III & 3 & 1.151 & $1.5 \mathrm{~mm}$ & 0.0022 \\
IV & 71 & 27.24 & $500 \mu \mathrm{m}$ & 0.0057 \\
V & 218 & 83.64 & $300 \mu \mathrm{m}$ & 0.0064 \\
VI & $2.9 \times 10^{7}$ & $1.113 \times 10^{7}$ & $1 \mu \mathrm{m}$ & 0.0094 \\
\hline \hline
\end{tabular}

\section{Particulate Contamination due to Shroud Deployment}

The shroud is deployed by inflating the gas bags in the forward part of the shroud. The enlarged bags open up the seam line and push the shrouds apart until the two halves are unlatched from their hinges at the base. There are two particulate sources during the deployment process: particles from the bag surface to the aperture window and particles generated due to ripping apart of the seam line. The issues are addressed as follows. 
The problem of particle resuspension and redistribution from the gas bag surface is treated similar to what discussed in the preceding section. As the gas bags are inflated to the fully extended position, the mean surface vibration caused by the pyroshock has to be determined. An accurate calculation of the surface vibration level can be made with a gas bag model and the pyroshock analysis (Ref. 5). For the present analysis, a level of $20 \mathrm{~g}$-rms is assumed.

The particles generated from the gas bag surfaces fall on the forecone. A uniform distribution of the particle over the forecone surface is again assumed. Because of the $15^{\circ}$ half-cone angle, the contribution to surface particle density is multiplied by $\cos \left(75^{\circ}\right)$. Table 7 summarizes the obscuration increases of the aperture surface from a range of gas bag surface cleanliness levels.

Table 7 Aperture window obscuration increase from gas bag operation.

\begin{tabular}{cc}
\hline \hline Gas bag surface Level & Obscuration increase, \% \\
\hline 750 & 0.1442 \\
700 & 0.1000 \\
600 & 0.0448 \\
500 & 0.0178 \\
400 & 0.0059 \\
300 & 0.0015 \\
\hline
\end{tabular}

A similar analysis is applied to calculate the obscuration increase of the aperture window due to seam line opening. The exact particle generation rate of the seam line opening is not available at the time of the analysis. However, the test result for the Centaur standard shroud separation system contamination was available (Ref. 6). In the longitudinal joint test the particle generation for the separation system using super-zip tube was measured. The test result is adopted here to show the potential effects of seam line opening during the shroud deployment. The predicted results are given in Table 8.

Table 8 Seam line opening induced aperture window obscuration increase.

\begin{tabular}{cccc}
\hline $\begin{array}{c}\text { Particle size } \\
\mu \mathrm{m}\end{array}$ & $\begin{array}{c}\text { Particle Count } \\
\text { \#/inch }\end{array}$ & $\begin{array}{c}\text { Converted } \\
\text { to \#/sq ft }\end{array}$ & $\begin{array}{c}\text { Percent } \\
\text { Obscuration }\end{array}$ \\
\hline $20-50$ & 680 & 11050 & 0.00762 \\
$50-100$ & 84 & 1365 & 0.00347 \\
$100-150$ & 12 & 195 & 0.00109 \\
$150-250$ & 6 & 98 & 0.00079 \\
$250-500$ & 1 & 16 & 0.00011 \\
$>500$ & 1 & 16 & 0.00017 \\
\hline Total & & & $0.01325 \%$ \\
\hline
\end{tabular}




\section{Other Particulate Contamination Considerations}

The contamination concerns from TLX line gas leakage was examined. Because the TLX line is located on the opposite side of the aperture window, and the shroud is deployed at zero angle of attack where there is no cross flow, the leakage, if any, will not cause any deposit on the aperture window. Therefore the TLX leakage is not a concern in the particulate contamination of the aperture window.

Questions were raised on the possibility that the particles on the aperture window will be scrubbed off by the shearing force after the protective shroud is jettisoned. An analysis was performed to address this problem (Ref. 7). The results show that at free stream Mach number equal to 6 , the shearing force is still less than the adhesion force of a $10-\mu \mathrm{m}$ particle on the surface at altitudes as low as $15 \mathrm{~km}$. The larger particles, e.g. $30 \mu \mathrm{m}$ or larger, may be removed by the shearing force at this altitude. At higher altitudes, because of low ambient density, the shearing force decreases further with the altitude. At $100 \mathrm{~km}$, the ambient density is five orders of magnitude lower than that at $15 \mathrm{~km}$, and no particles will be removed at this and higher altitudes. It is concluded that the shearing cleaning of the window surface is possible, but not effective.

The particulate contamination caused by the charred particles from aeroheating on the aperture window seal was not pursued because of lack of proper data. This remains an open issue at this time, though its effects are expected to be small.

Operation of the divert jets near the terminal phase of the flight is a potential contamination concern. The plume interacts with the flowfield and induces boundary layer flow separation. The circulation of flow in the separated region may carry the combustion products and surface particles forward to deposit on the aperture window. This problem is out of the scope of this study and is not discussed here.

\section{SCATTERING FROM SURFACE PARTICLES}

The LMSC Mie scattering/diffraction (RADSCA) model (Refs. 8 and 9) was used to compute the BSDF of the surface particles. The RADSCA code was verified by comparing its results to those generated with a published scattering model, PBHMIED (Refs. 10 and 11). Using the particle distribution of a Level 500 surface, the BSDF is computed with both codes.

Figure 1 shows a comparison between the BSDF calculated from PBHMIED, indicated with the solid line, using spherical particles and the results from the Mie scattering code for the Level 500 surface, noted with circles and squares. The coordinate is in $\left|\beta-\beta_{0}\right|$, where $\beta$ is $\sin \theta$ and $\theta$ is the scatter angle. $\beta_{0}$ is the sine of the incident angle $\theta_{0}$. The results using spherical particles from RADSCA agree well with the PBHMIED results. The results using cylindrical particles from RADSCA shows significant deviation at large scatter angles where $\theta$ is greater than $20^{\circ}$. Since the BSDF depends strongly on the wavelength and the particle shape, the deviation in BSDF caused by a different particle shape is not unexpected.

\section{BSDF Predictions}

For a window with the cleanliness level specified on both sides, the number of surface particles is assumed to be the sum of the numbers of both sides. For a clean window, which is at Level 300 for both sides at the beginning of life, the particle counts are doubled that of a Level 300 surface. To see the effects of the added particles on the inside of the window, the BSDF for the window are computed. Figure 2 shows the BSDF of three window levels for $3 \mu \mathrm{m}$ wavelength. 
The three BSDF curves correspond to the window of Level 300 on both sides, Level 300 on one side and Level 400 on the other side, and Level 400 on both sides. If the scattering limit is $10^{-3}$ at $20^{\circ}$, then only the window at Level 300 on both side will meet the requirement. The effects of window thickness and light refraction/transmission were not included in these calculations.

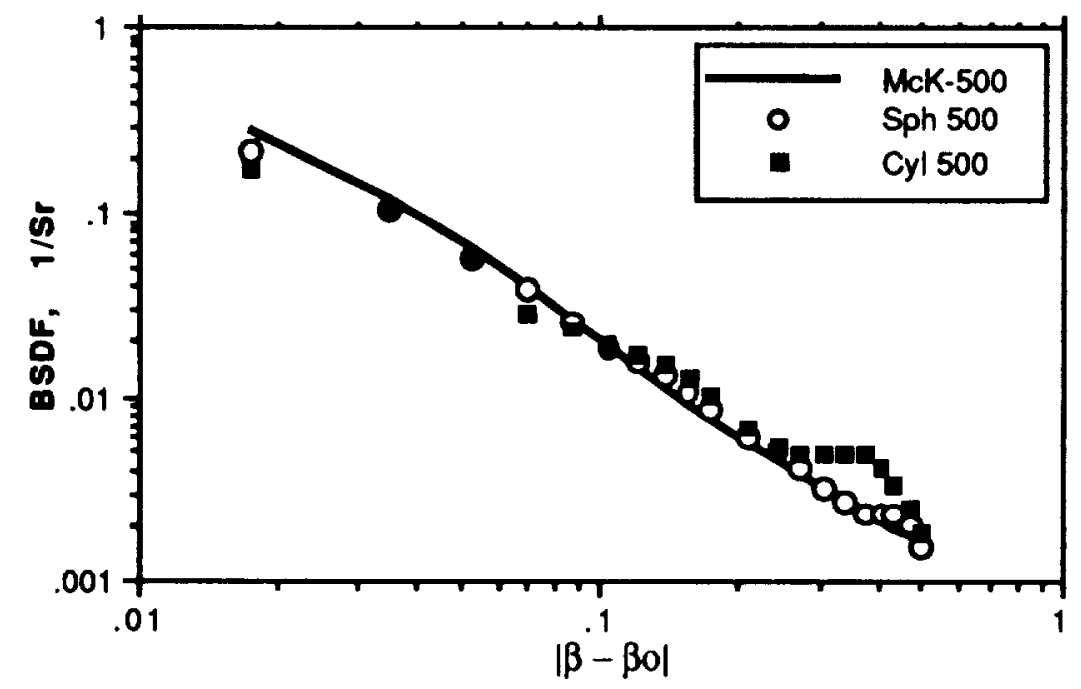

Fig. 1 Comparison of sphere-cylinder model for CL $500\left(\lambda=3 \mu \mathrm{m}, \theta_{\mathrm{O}}=0^{\circ}\right)$. The solid line indicates the results from PBHMIED with spherical particles.

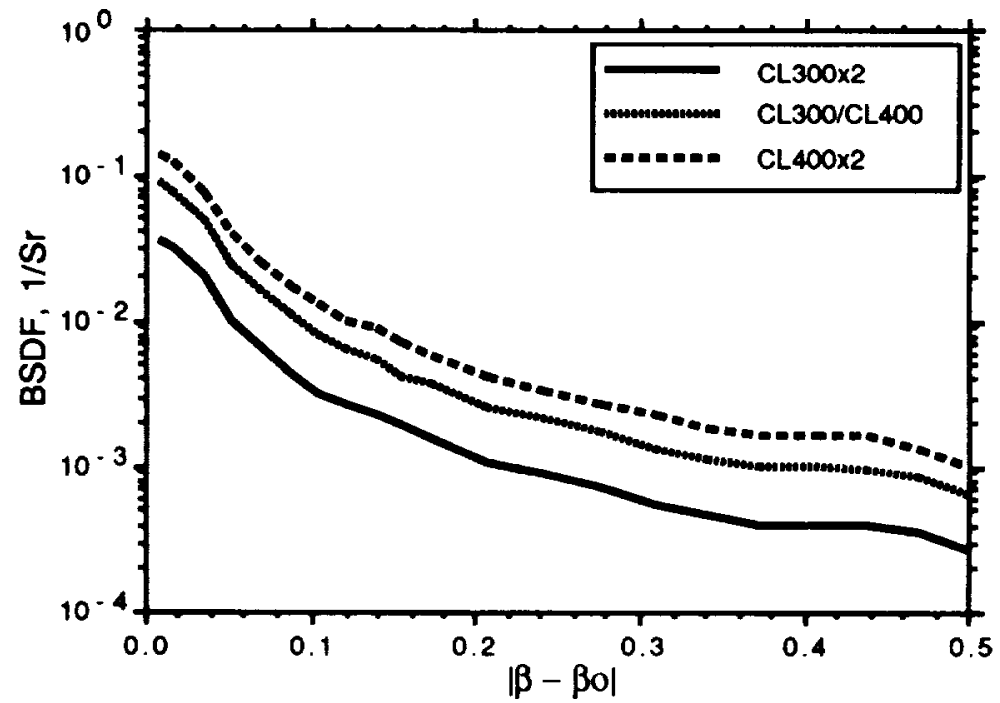

Fig. 2 The BSDF for the aperture window counted both sides.

For a practical analysis, let the window be at Level 300 on both sides initially. This level is attained by cleaning the window at the beginning of the manufacturing process to Level 100 and allowing for particle fallout during integration and tests.

As the vehicle goes through the events during ascent flight, additional particles will fall on the window due to vibroacoustic-induced redistribution, ingestion of foreign particles (of volcano 
dusts, etc.), and deployment (which generates particles from unzipping the seam and popping of the gas bags). Taking these effects into consideration and assuming that the inside surface of the window is still at Level 300 , the BSDF variation of the combined particle count is shown in Fig. 3 below. The solid line in the figure indicates the BSDF contributed from the outside window surface alone after accounting for the ascent flight effects. The dotted line shows the combined BSDF of the outside surface plus the inside surface at Level 300 . The figure shows that the scattering level of the window is less than $10^{-3}$ at $20^{\circ}$ and meets the straylight requirement.

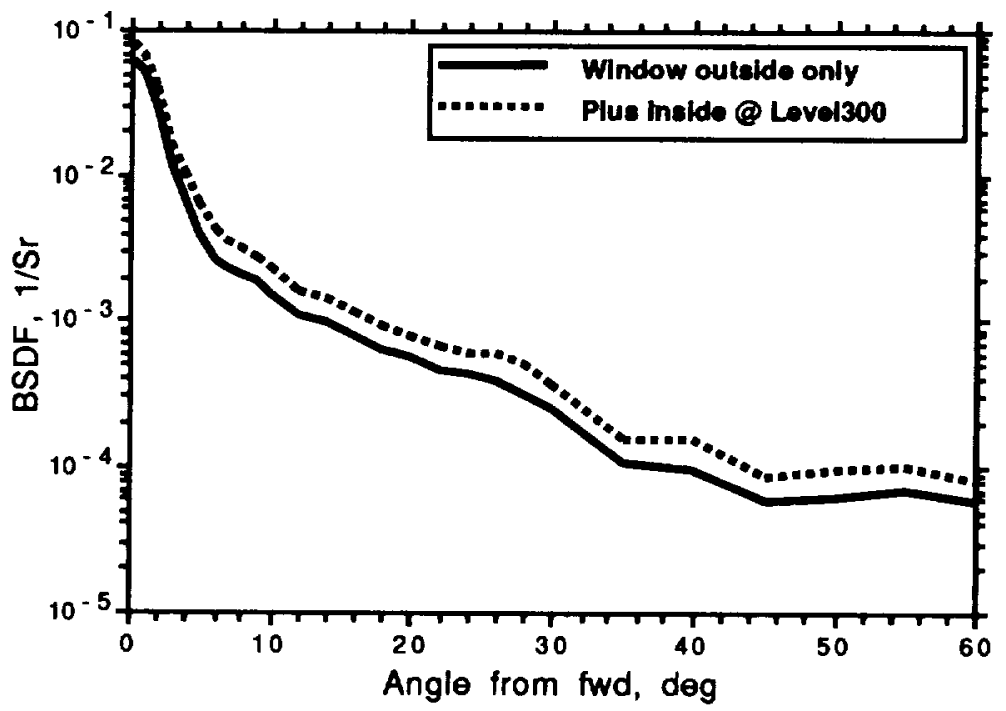

Fig. 3 The BSDF of the aperture window after going through ascent events and deployment for $\lambda=3 \mu \mathrm{m}$.

As the vehicle is subjected to the ascent vibroacoustic environment, the particles on the inside surface of the aperture window may also be resuspended. Because of the direction of acceleration vector, the resuspended particles are likely to be removed from the window without replenishment, thus leading to a cleaner inside surface than its initial cleanliness. Taking this effect into consideration, the resulting BSDF is shown in Fig. 4. In the figure, the BSDF of the aperture window for 3 to $5 \mu \mathrm{m}$ wavelengths are shown. It is seen that the values of BSDF for a given set of wavelengths vary with scatter angle. At $0^{\circ}$ the BSDF of the short wavelength (i.e. $3 \mu \mathrm{m}$ ) is the highest among the three, while at $25^{\circ}$ the $4 \mu \mathrm{m}$ value supersedes and so on. All three BSDF values at $20^{\circ}$ scatter angle are less than $10^{-3}$, and meet the straylight requirement.

\section{Scattering Caused by Surface Roughness}

The scattering caused by surface roughness is expressed in term of Total Integrated Scattering (TIS). The TIS can be measured with a simple instrument and correlated with the root mean square (rms) roughness of the surface (Ref. 12). The relation to convert the TIS to sample rms roughness is obtained from scalar scattering theory. The following formula can be used to calculate the TIS with given rms roughness $\mathrm{d}$ in $\AA$ and the wavelength $\lambda$ in $\AA$.

$$
\text { TIS }=(4 \pi d / \lambda)^{2}
$$


If the window surface has a rms roughness of $6 \AA$ and the wavelength is assumed to be 4 $\mu \mathrm{m}$, the TIS of the bare window is calculated as $3.6 \times 10^{-6}$, which is negligible comparing to the scattering contributed by the surface particles.

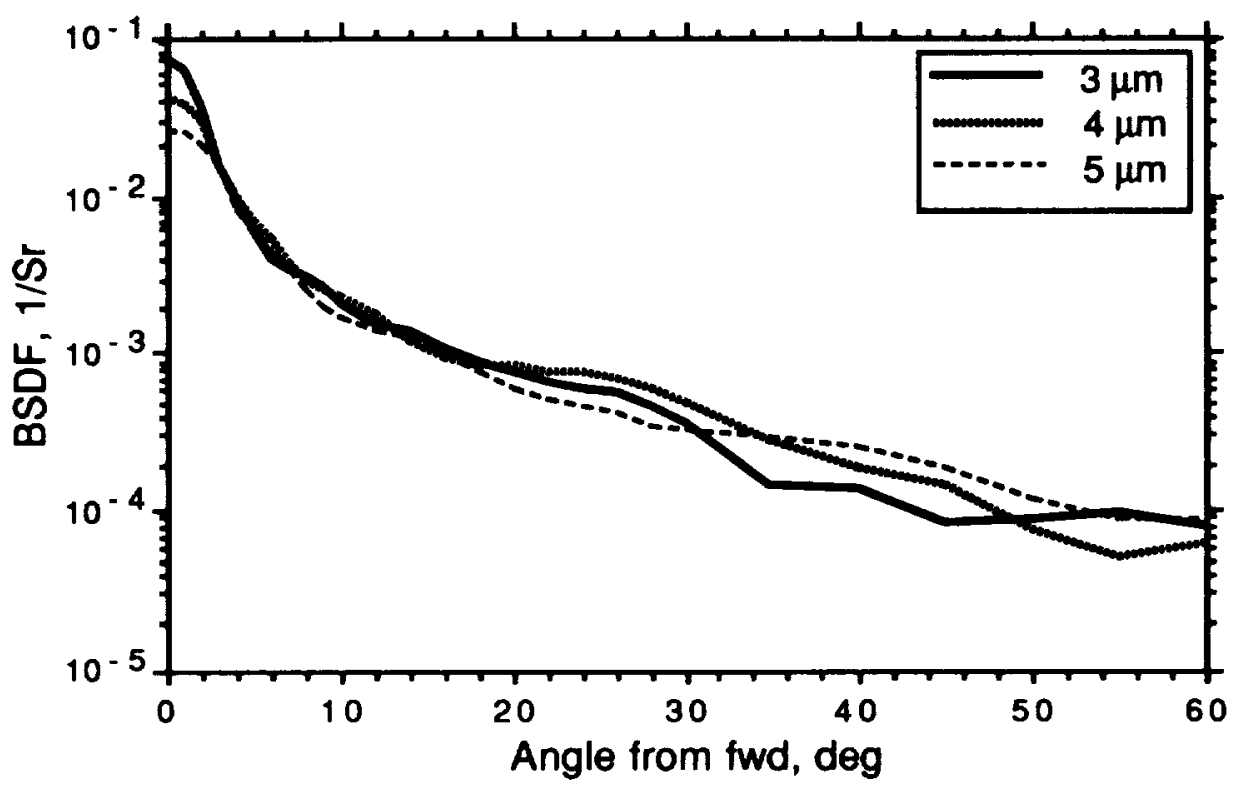

Fig. 4 The final BSDF for the aperture window for wavelengths of $3 \mu \mathrm{m}, 4 \mu \mathrm{m}$, and $5 \mu \mathrm{m}$.

\section{CONCLUSIONS}

A methodology to analyze the particulate contamination on the aperture window has been developed and presented in this paper. The analytical approach begins with a correlation between the specification of surface cleanliness level and the surface obscuration ratio to the surface particle densities in the size bins.

The particulate contamination increments contributed by the events during ascent and flight phases have been identified and predicted quantitatively in the analysis. The events include Vibroacoustic-induced particle redistribution, ingestion of particles during ascent, and the particles generated by shroud deployment.

The effects of particulate contamination on the aperture window are expressed in terms of BSDF. The software tool used to compute the BSDF was verified by comparison with the results from a published tool. The result shows the BSDF from the final particulate contamination on the aperture window at the end of mission. In the sample case used in the study, the predicted BSDF level meets the prescribed requirement. 


\section{REFERENCES}

1. P. T. Ma, M. C. Fong, and A. L. Lee, "Surface Particle Obscuration and BRDF Prediction," Scattering from Optical Components, SPIE Proceedings Vol. 1165, edited by J. C. Stover, SPIE Paper No. 1165-33, 1989.

2. P. T. Ma, "An Improved Particle Area Coverage Calculation Method," LMSC-SSD TSS-371, July 5, 1989.

3. Y. A. Lee, W. Hendricks, D. Park, et al., "Vibroacoustic Payload Environment Prediction System (VAPEPS)," NASA CR-166823, 1984.

4. A. Klavins and A. L. Lee, "Spacecraft Particulate Contaminant Redistribution," Optical System Contamination: Effects, Measurement, Control, SPIE Proceedings Vol. 777, edited by A. P. Glassford, Paper No. 777-27, 1987.

5. Y. A. Lee and W. Hendricks, "Pyroshock Analysis and Test of Composite Satellite Structure," DGLR/AIAA 92-02-167, 1992.

6. D. D. Smith, "Centaur Standard Shroud Separation System Contamination Test Report," LMSC-D466881, 10 September 1976.

7. C. Fan, "Shear Force Cleaning for Surface Particulate," LMSC-SSD TSS-478A, 3 June 1993.

8. E. C. Markovitz, "A Computer Code to Calculate the Straylight due to Particulate Contaminants on a Surface," LMSC-SSD TSS-301, May 19, 1986.

9. M. C. Fong, "Spacecraft Contamination Modeling Capability at LMSC - A Comprehensive Overview," LMSC-SSD TSS-473, November 18, 1992.

10. P. R. Spyak, "A Cryogenic Scatterometer and Scatter from Particulate Contaminants on Mirror," Univ. of Arizona Dissertation, 1990, also Optical Engineering, August 1992, Vol. 31, No. 8, pp.1746-1784.

11. P. R. Spyak and W. L. Wolfe, "Cryogenic Scattering Measurements," Stray Light and Contamination in Optical System, SPIE Vol. 967, 1988, pp.144-158.

12. J. C. Stover, Optical Scattering - Measurement and Analysis, McGraw-Hill, 1990, pp.87. 
